\title{
Opioid and/or Psychotropic Use Increases Intraprocedural Sedation Drug Requirements
}

\author{
${ }^{1}$ Department of Radiology, University of Kentucky College of \\ Medicine, Lexington, Kentucky, United States \\ ${ }^{2}$ Department of Radiology, University of Kentucky, Lexington, \\ Kentucky, United States \\ ${ }^{3}$ Division of Interventional Radiology, Department of Radiology, \\ University of Kentucky, Lexington, Kentucky, United States \\ J Clin Interv Radiol ISVIR 2021;6:23-27.
}

Sreeja Sanampudi ${ }^{1}$ Ravi Jayavarapu ${ }^{2}$ Trae Brooks ${ }^{1, \odot}$ Driss Raissi ${ }^{3}$

\begin{abstract}
Address for correspondence Sreeja Sanampudi, MD, University of Kentucky College of Medicine, MN150, Lexington, KY 40506, United States (e-mail: sreeja.sanampudi@gmail.com).
\end{abstract}

\begin{abstract}
Objectives Acute pain management in opioid users can be challenging in the perioperative period. This study focuses on whether use of opioids increases sedation medication requirements in patients undergoing port placement under moderate sedation. Materials and Methods A retrospective review was performed on all patients undergoing port placement between June 1, 2017, and June 30, 2019. Exclusion criteria included receiving general anesthesia, no sedation, and errors in data entry. Data collection included demographics, use of opioids, benzodiazepines, antidepressants, tobacco, alcohol, and sedation data.

Results Opioid, benzodiazepine, and selective serotonin reuptake inhibitors (SSRIs)/serotonin-norepinephrine reuptake inhibitors (SNRIs) use was significantly associated with higher sedation drug dose requirements. Patients using opioids required $10.5 \%$ higher doses of midazolam compared with nonusers. Benzodiazepine users required $16.3 \%$ additional dosage of midazolam than nonusers. Finally, patients on SSRIs/SNRIs medications required $11.8 \%$ higher midazolam dosing when compared with nonusers.

Keywords

- sedation

- opioids

- benzodiazepines

Conclusion Anticipating higher needs of sedation medications during procedures in patients with history of psychotropic agents use can allow for more effective sedation and patient satisfaction. More patient and provider awareness is needed on this topic, as health care policy is moving toward value-based healthcare, with patient satisfaction surveys being one of its indicators.
\end{abstract}

\section{Introduction}

Opioids are often used perioperatively-short term in acute postprocedure period or long term in patients with cancer related pain or palliative care. ${ }^{1}$ In the past several decades, there has been an increase in opioid use for chronic noncancer-related pain; this trend has only recently started to decrease with improved utilization of nonopioid, nonpharmacologic interventions, and implementation of clinical guidelines in using opioids. ${ }^{1-4}$ Overall opioid prescriptions have increased from 2006 and peaked in 2012 at more than 255 million dispensed prescriptions. It downtrended from 2012 with 168 million prescriptions dispensed in 2018. ${ }^{5}$ With use of opioids, there is concern for tachyphylaxis, dependence, opioid-induced hyperalgesia, and even opioid-related respiratory depression and death. ${ }^{6,7}$ Opioid tolerance, as defined by FDA, occurs when an individual takes $\geq 1$ week of the following agents and doses: $30 \mathrm{mg}$ oral oxycodone/day, published online May 18, 2021
DOI https://doi.org/ $10.1055 / \mathrm{s}-0041-1729469$ ISSN 2457-0214

\footnotetext{
(C) 2021. Indian Society of Vascular and Interventional Radiology.

This is an open access article published by Thieme under the terms of the Creative Commons Attribution-NonDerivative-NonCommercial-License, permitting copying and reproduction so long as the original work is given appropriate credit. Contents may not be used for commercial purposes, or adapted, remixed, transformed or built upon. (https://creativecommons.org/licenses/by-nc-nd/4.0/).

Thieme Medical and Scientific Publishers Pvt. Ltd. A-12, 2nd Floor,

Sector 2, Noida-201301 UP, India
} 
$25 \mathrm{mcg}$ transdermal fentanyl/hour, $60 \mathrm{mg}$ oral morphine/day, or an equivalent dose of a different opioid. ${ }^{8}$ Therefore, even short courses of opioids can result in tolerance with patients requiring higher doses to achieve similar analgesic effect. ${ }^{7}$ This pattern has been seen in patients taking opioids for both cancer and noncancer-related pain. ${ }^{9}$

Pain management during perioperative period can be challenging in patients who have history of prescription opioid use, opioid misuse, or even those on opioid agonist/antagonist regimens for withdrawal and cessation. ${ }^{6}$ Different levels of sedation ranging from minimal sedation to general anesthesia need to be performed, based on procedure type to provide adequate sedation and analgesia during procedures. ${ }^{6,10}$ Moderate sedation is used frequently for minimally invasive procedures including endoscopies, bronchoscopies, port placements, central line placements, percutaneous biopsies (i.e., liver, kidney, lung), and drain placements, etc. ${ }^{10-12}$ Benzodiazepines and opioids are used during moderate sedation, wherein patients are easily arousable to verbal and tactile stimuli and are able to protect their airway and maintain spontaneous ventilation. ${ }^{10}$ Benzodiazepines and opioids have several therapeutic effects, and the anxiolytic effects of benzodiazepines allows for decreased perception of pain by working synergistically with opioid and nonopioid analgesic medications..$^{10,13,14}$ Adequate sedation allows for timely and ease in procedure completion while minimizing patient discomfort. ${ }^{10}$

Higher sedation requirements in patients with history of opioid and alcohol use disorders has been documented during colonoscopies and mechanical ventilation. ${ }^{11,15}$ Other factors contributing to higher sedation requirements include anxiety, agitation, and pre-existing high pain sensitivity; some of these patients even have a history of concomitant use of benzodiazepines and antidepressants. ${ }^{11,16}$

The purpose of this study is to evaluate whether use of opioids would increase sedation medication requirements in patients undergoing minimally invasive procedures under moderate sedation at a single tertiary care center. We elected central venous port placement as an index minimally invasive procedure to decrease procedural heterogeneity.

\section{Materials and Methods}

\section{Patient Selection and Data Collection}

Following approval from local Institutional Review Board, a retrospective study was performed on all the patients who had port placement between June 1, 2017, and June 30, 2019 at a single tertiary care center. Informed consent was obtained from all patients included in the study prior to performing procedure and data collection. A total of 430 different instances of port placement occurred during this time period. Patients with known history of illicit opioid use and patients who had no sedation or required general anesthesia were excluded from the current study. In addition, cases with errors in data entry at time of procedure were also excluded. Based on these criteria, a total of 27 cases were excluded.

For the remaining 403 patients, electronic medical records were reviewed for following data collection: patient demographics, use of tobacco, alcohol use, and sedation data (amounts of midazolam, fentanyl, and diphenhydramine). A procedural nurse administered moderate sedation medications during port placement and a pain scale (1-10) goal of $<4$ was used as the target for adequate sedation. All the providers had at least 3 years of postinterventional radiology fellowship experience. The following definitions were used for opioids and other psychotropic substances. Use of opioids was defined as prescribed opioids for $\geq 1$ week within the last 3 years prior to port placement. Additional data collected included use of benzodiazepines (defined as current or history of use of benzodiazepine products for at least 7 days within 5 years prior to date of port placement), use of typical antidepressants (defined as current or history of use of selective serotonin reuptake inhibitors [SSRIs] and serotonin-norepinephrine reuptake inhibitors [SNRIs] for at least 7 days within 5 years prior to date of port placement), and any documented history of use of pregabalin/gabapentin.

\section{Statistical Analysis}

Statistical analysis was performed using SAS software version 9.4 (SAS Institute Inc., Cary, NC, USA). $t$-tests were used to identify average doses of midazolam and fentanyl used in patients with use of opioids and other psychotropic substances including benzodiazepines, gabapentin/pregabalin, and antidepressants. Overall mean, standard deviations, and $p$ values were calculated using SAS software. Significance was indicated by a $p$ value of $\leq 0.05$.

\section{Results}

Of the 403 remaining port placements, the following were the patient demographics: $44 \%$ were male, $92 \%$ Caucasian, $7 \%$ African-American and 1\% Asian; $18 \%$ had history of alcohol abuse and 53\% had history of tobacco use. Of the 403 cases, $185 / 403$ (46\%) had used opioids for at least 1 week in the 3 years prior to port placement, 81/403 (20\%) had used benzodiazepines for at least 1 week in the 5 years prior to port placement, 81/403 (20\%) of the patients had used antidepressants for at least 7 days in the 5 years prior to port placement, and $44 / 403$ (11\%) had used gabapentin/pregabalin.

Statistical analysis was performed using SAS software. Patients who were opioid users needed $10.5 \%$ significantly higher doses of midazolam when compared with nonusers ( $p$ value $=0.04$ ), and these patients also required $7.9 \%$ higher doses of fentanyl compared with nonusers; however, the latter results were not statistically significant ( $p$ value $=0.09$ ). Patients who had history of use of benzodiazepines required $11.3 \%$ significantly higher doses of fentanyl and $16.3 \%$ significantly higher doses of midazolam when compared with nonusers $(p$ value $=0.05$ and 0.01 , respectively). Finally, patients with history of antidepressant use required $11.9 \%$ significantly higher doses of midazolam and $7.8 \%$ higher doses of fentanyl when compared with nonusers, however, the latter was not statistically significant ( $p$ values 0.05 and 0.17 , respectively). Patients who were users of pregabalin/gabapentin required higher doses of fentanyl and midazolam when compared with nonusers; 
Table 1 Demonstrates the average midazolam and fentanyl doses required in patients with use of opioids and other psychotropic agents

\begin{tabular}{|c|c|c|c|c|}
\hline Psychotropic medication class & Midazolam (mg) & $p$ value & Fentanyl (mcg) & $p$ value \\
\hline Opioids: & & \multirow[t]{3}{*}{0.04} & & \multirow[t]{3}{*}{0.09} \\
\hline Users: & $2.7 \pm 1.3$ & & $123 \pm 60$ & \\
\hline Nonusers: & $2.5 \pm 1.1$ & & $114 \pm 48$ & \\
\hline Benzodiazepines: & & \multirow[t]{3}{*}{0.01} & & \multirow[t]{3}{*}{0.05} \\
\hline Users: & $2.9 \pm 1.4$ & & $129 \pm 54$ & \\
\hline Nonusers: & $2.5 \pm 1.6$ & & $116 \pm 54$ & \\
\hline Antidepressants: & & \multirow[t]{3}{*}{0.05} & & \multirow[t]{3}{*}{0.17} \\
\hline Users: & $2.8 \pm 1.2$ & & $126 \pm 52$ & \\
\hline Nonusers: & $2.5 \pm 1.2$ & & $117 \pm 54$ & \\
\hline Pregabalin/gabapentin: & & \multirow[t]{3}{*}{0.81} & & \multirow[t]{3}{*}{0.62} \\
\hline Users: & $2.6 \pm 1.5$ & & $122 \pm 63$ & \\
\hline Nonusers: & $2.6 \pm 1.2$ & & $118 \pm 53$ & \\
\hline
\end{tabular}

Note: $p$ values obtained by performing $t$-tests through SAS software are also represented in this table.

however, these results were not statistically significant. - Table 1 demonstrates average dose of midazolam and fentanyl with associated standard deviations and $p$ values in patients with use of opioids and other psychotropic agents.

\section{Discussion}

After heart disease, cancer is the most common cause of death with an estimated approximately 600,000 deaths expected in 2020. ${ }^{17}$ However, given advances in treatment, earlier detection of disease, improvement in screening modalities, and decrease in risk factors such as smoking, cancer death continues to decrease. This has resulted in greater number of cancer survivors, thus resulting in a greater number of patients managing cancer-related pain on a day-to-day basis., ${ }^{9,17}$ Opioids continue to be one of the primary mainstays in treatment of severe cancer-related pain when nonopioid interventions have failed or to augment these interventions for breakthrough pain. In addition, there has been an overall increase in prescription of benzodiazepines over the past several decades. One study reported a $67 \%$ increase in number of individuals filling benzodiazepine prescriptions from 1996 to $2013 .{ }^{18}$ During the same time period, the study noted three times increase in the quantity of benzodiazepines filled due to increase in dosage, number of prescriptions, duration of prescriptions, or a combination of these factors. ${ }^{18}$

Tachyphylaxis, physical dependence, opioid-induced hyperalgesia, addiction, and overdose continue to be concerning factors with use of opioids. A single dose may be sufficient to develop tolerance for some opioid effects; however, in most cases, development of tolerance depends on duration, dose, and type of opioid utilized. ${ }^{3}$ Tolerance to effects of analgesia and euphoria occur earlier than effects of respiratory depression; thus, the increasing concern for overdose when dose escalation occurs. ${ }^{3}$ It is believed that dose escalation up to 10 times initial dose may be required for similar analgesic effects. ${ }^{3}$
However, following cessation of opioid use, effects of tolerance and dependence resolve within days to weeks, depending on strength, duration and type of opioid used. ${ }^{3}$

Various studies have looked at sedation in patients with history of substance use disorders in various settings including endoscopies, mechanically ventilated patients, and dental procedures. ${ }^{11,15,19}$ Patel et al demonstrated that during sedation for colonoscopies, opioid users require significantly higher doses of fentanyl (by 23\%) and midazolam (by 24\%) compared with patients with alcohol use and control groups. ${ }^{11}$ This is consistent with findings from this current study, and it demonstrates significantly increased requirement of midazolam by $10.5 \%$ in patients who are opioid users in comparison to nonusers during port placement. These patients also had required 7.9\% increase in fentanyl dosing; however, these results were not significant at the 95\% confidence interval. Another study demonstrated that during mechanical ventilation in intensive care unit, patients with alcohol use disorder needed greater amounts of sedatives and opioids to achieve similar degree of sedation than their nonalcoholic counterparts. ${ }^{15}$ Cook et al also demonstrated that greater amounts of diazepam are required to achieve similar levels of sedation in patients using alcohol and benzodiazepines even at low doses when these patients are undergoing elective dental and endoscopic procedures. ${ }^{19}$ These results are similar to the current study. Patients who had history of benzodiazepine use required $11.3 \%$ significantly higher doses of fentanyl and $16.3 \%$ significantly higher doses of midazolam when compared with nonusers. Tolerance has been attributed to increased requirements of sedatives in patients with history of benzodiazepine use. ${ }^{19,20}$ Sedative and hypnotic effects of benzodiazepines develop tolerance earlier as compared with other therapeutic effects of benzodiazepines, and tolerance occurs much faster with shorter half-life agents. ${ }^{20,21}$ However, benzodiazepines appear to have continued efficacy against anxiolysis even with long-term use, and some studies have attributed this as 
reason for lack of dose escalation in chronic benzodiazepine users. ${ }^{20,22}$ Overall, both opioids and benzodiazepines augment each other's effects during sedation, and the increase in dose requirements during perioperative period is likely due to tolerance exhibited to both agents. ${ }^{10,11,20}$

To our knowledge, this is the first study that demonstrated not only increased midazolam requirements in patients with history of prescription benzodiazepine use, but also significantly increased midazolam requirements in patients with history of prescription antidepressant use (SSRIs and SNRIs) in patients undergoing port placement. In addition, these patients also required increased doses of fentanyl during port placement when compared with non-users, however, these results were not significant at the $95 \%$ confidence interval. The exact mechanism through which antidepressant users require higher benzodiazepines is unclear. However, it is likely multifactorial, with a combination of biochemical changes and variable neurotransmitter availability in the central nervous system (CNS) caused by multiple psychotropic medications and presence of somatic and psychological factors during the perioperative period. ${ }^{23-25}$ In our study, anxiety and mood changes during the procedure likely potentiate pain perception, which subsequently results in higher midazolam and fentanyl doses in patients with history of prescription antidepressant use. Patients with preoperative depression and anxiety reported having greater amounts of postoperative pain and resultant increase in opioid use. ${ }^{25,26}$ One study performed psychological assessment (that included patient health questionnaire among other questionnaires) in all patients prior to oropharyngeal surgery. The study indicated that significantly more opioids were needed for higher postoperative pain which, in turn, was correlated to psychological anticipation of higher pain and/or with higher degree of clinical depression. ${ }^{25}$ Although these studies looked at postoperative pain, similar rationale can be extrapolated to the intraoperative period.

To our knowledge, this is the first study that has looked at increased sedation requirements during a discrete minimally invasive procedure performed under moderate sedation in patients with known history of prescription opioid use. Central venous ports were chosen as an ideal index procedure to limit procedural heterogeneity such as anatomical location, procedural duration, and procedure -related pain. Port placement is a relatively standardized procedure that is short in duration, involves minimal intraprocedural discomfort, and has little to no postprocedural pain.

Approximately, 1.8 million new cases of cancer are expected to be diagnosed in 2020 in United States, a significant portion of whom will require central venous access devices (i.e., port) for infusion of chemotherapy. ${ }^{17}$ Therefore, identifying patients who have been on opioids for $\geq 1$ week can allow interventionalists to provide optimal preprocedural sedation planning.

This study does have some limitations. It is a retrospective study at a single institution, and data was collected on a single procedure type (port placement). Additional limitations include variable strengths and durations of use of opioids and other psychotropic agents (ranging from 1 week to several months). Also, patient-reported pain scores can be highly subjective and would be difficult to account for. Furthermore, nurses and/or physicians were not blinded to patient's medical history of opioid and/or psychotropic use, potentially introducing an element of bias.

\section{Conclusion}

Patients with history of opioid use require significantly higher sedation drug doses compared with nonusers, and similar trends can be seen in patients with history of use of benzodiazepines and antidepressants. Anticipating higher requirements of these medications in these patients can allow planning for other alternatives for analgesia/anesthesia, increase patient satisfaction, and allow timely completion of minimally invasive procedures performed under moderate sedation. More patient and provider awareness are needed on this topic, as health care policy has been moving more and more toward value-based health care, with patient satisfaction surveys being one of its indicators.

\section{Conflict of Interest}

None declared.

\section{References}

1 Chou R. 2009 Clinical Guidelines from the American Pain Society and the American Academy of Pain Medicine on the use of chronic opioid therapy in chronic noncancer pain: what are the key messages for clinical practice? Pol Arch Med Wewn 2009;119(7-8):469-477

2 Dowell D, Haegerich TM, Chou R. CDC Guideline for Prescribing Opioids for Chronic Pain - United States, 2016. [published correction appears in MMWR Recomm Rep. 2016;65(11):295] MMWR Recomm Rep 2016;65(1):1-49

3 Volkow ND, McLellan AT. Opioid abuse in chronic painMisconceptions and mitigation strategies. $\mathrm{N}$ Engl J Med 2016;374(13):1253-1263

4 Volkow N, Benveniste H, McLellan AT. Use and misuse of opioids in chronic pain. Annu Rev Med 2018;69:451-465

5 U.S. Opioid Prescribing Rate Maps. CDC. Available at https:// www.cdc.gov/drugoverdose/maps/rxrate-maps.html. Accessed September 25, 2020

6 Adesoye A, Duncan N. Acute pain management in patients with opioid tolerance. US Pharmacist 2017;42(3):28-32

7 Mitra S, Sinatra RS. Perioperative management of acute pain in the opioid-dependent patient. Anesthesiology 2004;101(1):212-227

8 FDA. Extended-release (ER) and long-acting (LA) opioid analgesics Risk Evaluation and Mitigation Strategy (REMS). https:// www.fda.gov/media/83883/download. Accessed September 25,2020

9 Datto CJ, Hu Y, Wittbrodt E, Fine PG. Opioid utilization patterns among patients with cancer and non-cancer pain. J Opioid Manag 2019;15(1):11-18

10 Moran TC, Kaye AD, Mai AH, Bok LR. Sedation, analgesia, and local anesthesia: a review for general and interventional radiologists. Radiographics 2013;33(2):e47-e60

11 Patel R, Clayton S, Quintero E, Gill J. chronic opioid users are more difficult to sedate than alcoholics and controls. South Med J 2015;108(12):744-747

12 José RJ, Shaefi S, Navani N. Sedation for flexible bronchoscopy: current and emerging evidence. Eur Respir Rev 2013;22(128):106-116 
13 Soyka M. Treatment of benzodiazepine dependence. N Engl J Med 2017;376(12):1147-1157

14 Lader M. Benzodiazepine harm: how can it be reduced? Br J Clin Pharmacol 2014;77(2):295-301

15 de Wit M, Wan SY, Gill S, et al. Prevalence and impact of alcohol and other drug use disorders on sedation and mechanical ventilation: a retrospective study. BMC Anesthesiol 2007;7:3

16 Osborn TM, Sandler NA. The effects of preoperative anxiety on intravenous sedation. Anesth Prog 2004;51(2):46-51

17 Cancer Facts \& Figures 2020. American Cancer Society. Available at https://www.cancer.org/content/dam/cancer-org/research/cancer-facts-and-statistics/annual-cancerfacts-and-figures/2020/cancer-facts-and-figures-2020.pdf. Accessed September 25, 2020

18 Bachhuber MA, Hennessy S, Cunningham CO, Starrels JL. Increasing benzodiazepine prescriptions and overdose mortality in the United States, 1996-2013. Am J Public Health 2016;106(4):686-688

19 Cook PJ, Flanagan R, James IM. Diazepam tolerance: effect of age, regular sedation, and alcohol. Br Med J (Clin Res Ed) 1984;289(6441):351-353

20 Vinkers $\mathrm{CH}$, Olivier B. Mechanisms underlying tolerance after long-term benzodiazepine use: a future for subtypeselective GABA (A) receptor modulators? Adv Pharmacol Sci 2012;2012:416864
21 Bateson AN. Basic pharmacologic mechanisms involved in benzodiazepine tolerance and withdrawal. Curr Pharm Des 2002;8(1):5-21

22 Willems IA, Gorgels WJ, Oude Voshaar RC, Mulder J, Lucassen PL. Tolerance to benzodiazepines among long-term users in primary care. Fam Pract 2013;30(4):404-410

23 Attri JP, Bala N, Chatrath V. Psychiatric patient and anaesthesia. Indian J Anaesth 2012;56(1):8-13

24 Taylor C, Fricker AD, Devi LA, Gomes I. Mechanisms of action of antidepressants: from neurotransmitter systems to signaling pathways. Cell Signal 2005;17(5):549-557

25 Suffeda A, Meissner W, Rosendahl J, Guntinas-Lichius O. Influence of depression, catastrophizing, anxiety, and resilience on postoperative pain at the first day after otolaryngological surgery: A prospective single center cohort observational study. Medicine (Baltimore) 2016;95(28):e4256

26 Pan X, Wang J, Lin Z, Dai W, Shi Z. Depression and anxiety are risk factors for postoperative pain-related symptoms and complications in patients undergoing primary total knee arthroplasty in the United States. J Arthroplasty 2019;34(10):2337-2346 